\title{
General Solution and Stability of Quattuordecic Functional Equation in Quasi $\beta$-Normed Spaces
}

\author{
K. Ravi1, J. M. Rassias², S. Pinelas ${ }^{3}$, S. Suresh ${ }^{4}$ \\ ${ }^{1}$ Department of Mathematics, Sacred Heart College, Tirupattur, Tamil Nadu, India \\ ${ }^{2}$ Pedagogical Department E. E, Section of Mathematics and Informatics, National and Capodistrian University of Athens, \\ Athens, Greece \\ ${ }^{3}$ Departamen to de Cincias Exactas e Naturais, Amadora, Portugal \\ ${ }^{4}$ Research and Development Centre, Bharathiar University, Coimbatore, India \\ Email: shckravi@yahoo.co.in, jrassias@primedu.uoa.gr, sandra.pinelas@gmail.com, sureshs25187@gmail.com
}

How to cite this paper: Ravi, K., Rassias, J.M., Pinelas, S. and Suresh, S. (2016) General Solution and Stability of Quattuordecic Functional Equation in Quasi $\beta$-Normed Spaces. Advances in Pure Mathematics, 6, 921-941.

http://dx.doi.org/10.4236/apm.2016.612070

Received: September 28, 2016

Accepted: November 27, 2016

Published: November 30, 2016

Copyright $\odot 2016$ by authors and Scientific Research Publishing Inc. This work is licensed under the Creative Commons Attribution International License (CC BY 4.0).

http://creativecommons.org/licenses/by/4.0/

\section{Abstract}

In this paper, we introduce the following quattuordecic functional equation

$$
\begin{aligned}
& f(x+7 y)-14 f(x+6 y)+91 f(x+5 y)-364 f(x+4 y)+1001 f(x+3 y) \\
& -2002 f(x+2 y)+3003 f(x+y)-3432 f(x)+3003 f(x-y)-2002 f(x-2 y) \\
& +1001 f(x-3 y)-364 f(x-4 y)+91 f(x-5 y)-14 f(x-6 y)+f(x-7 y) \\
& =14 ! f(y),
\end{aligned}
$$

investigate the general solution and prove the stability of this quattuordecic functional equation in quasi $\beta$-normed spaces by using the fixed point method.

\section{Keywords}

Quattuordecic Functional Equation, Fixed Point Method, Hyers-Ulam Rassias Stability, Quasi- $\beta$-Normed Space

\section{Introduction}

The first stability problem concerning group homomorphisms was raised by Ulam [1] in 1940. He stated that if $\left(G_{1},.\right)$ is a group and let $\left(G_{2}, *\right)$ be a metric group with metric $d(.,$.$) : Given \epsilon>0$, does there exist a $\delta>0$ such that if a mapping $h: G_{1} \rightarrow G_{2}$ satisfies the inequality $d(h(x y), h(x) * h(y))<\epsilon$ for all $x, y \in G_{1}$, then there exists a homomorphism $H: G_{1} \rightarrow G_{2}$ with $d(G(x), H(x))<\epsilon$ for all $x \in G_{1}$ ?

The case of approximately additive functions was solved by D. H. Hyers [2] under the assumption that both $E_{1}$ and $E_{2}$ are Banach spaces. He stated that for $\epsilon>0$ and 
$f: E_{1} \rightarrow E_{2}$ such that $\|f(x+y)-f(x)-f(y)\| \leq \varepsilon$ for all $x, y \in E_{1}$, then there exists a unique additive mapping $T: E_{1} \rightarrow E_{2}$ such that $\|f(x)-T(x)\| \leq \varepsilon$ for all $x \in E_{1}$. This result is called Hyers-Ulam stability.

Hyers Theorem was generalized by Th. M. Rassias [3] for linear mappings by considering an unbounded Cauchy difference. The stability problem of several functional equations has been extensively investigated by a number of authors, and there are many interesting results concerning this problem [4]-[17].

Very recently the general solution and the stability of the quintic and sextic functional equation in quasi- $\beta$-normed spaces via fixed point method were discussed by [18]. The general solution, the stability of the septic and Octic functional equations, viz.

$$
\begin{aligned}
& f(x+4 y)-7 f(x+3 y)+21 f(x+2 y)-35 f(x+y)-35 f(x) \\
& -21 f(x-y)+7 f(x-2 y)-f(x-3 y)=5040 f(y)
\end{aligned}
$$

and

$$
\begin{aligned}
& f(x+4 y)-8 f(x+3 y)+28 f(x+2 y)-56 f(x+y)-70 f(x)-56 f(x-y) \\
& +28 f(x-2 y)-8 f(x-3 y)+f(x-4 y)=40320 f(y)
\end{aligned}
$$

in quasi- $\beta$-normed spaces were investigated by T. Z. Xu et al. [18].

J. M. Rassias and Mohamed Eslamian discussed the general solution of a Nonic functional equation

$$
\begin{aligned}
& f(x+5 y)-9 f(x+4 y)+36 f(x+3 y)-84 f(x+2 y)-126 f(x+y)-126 f(x) \\
& +84 f(x-y)-36 f(x-2 y)+9 f(x-3 y)-f(x-4 y)=9 ! f(y)
\end{aligned}
$$

and proved the stability of nonic functional equation [19] in quasi- $\beta$-normed spaces by applying the fixed point method.

A fixed point approach for the stability of Decic functional equation

$$
\begin{aligned}
& f(x+5 y)-10 f(x+4 y)+45 f(x+3 y)-120 f(x+2 y)-210 f(x+y)-252 f(x) \\
& +210 f(x-y)-120 f(x-2 y)+45 f(x-3 y)-10 f(x-4 y)+f(x-5 y)=10 ! f(y)
\end{aligned}
$$

in quasi- $\beta$-normed spaces was investigated by K. Ravi et al. [20].

Very recently, K. Ravi and Senthil Kumar discussed the undecic and duodecic functional equation and its stability in quasi- $\beta$-normed spaces.

In this paper, the authors are interested in finding the general solution and stability of Quattuordecic functional equation

$$
\begin{aligned}
& f(x+7 y)-14 f(x+6 y)+91 f(x+5 y)-364 f(x+4 y)+1001 f(x+3 y) \\
& -2002 f(x+2 y)+3003 f(x+y)-3432 f(x)+3003 f(x-y)-2002 f(x-2 y) \\
& +1001 f(x-3 y)-364 f(x-4 y)+91 f(x-5 y)-14 f(x-6 y)+f(x-7 y) \\
& =14 ! f(y)
\end{aligned}
$$

where $14 !=87178291200$ in quasi- $\beta$-normed spaces by using fixed point method.

The functional Equation (1) is called Quattourdecic functional equation because the function $f(x)=K x^{14}$ satisfies the Equation (1). 
In Section 2, we have given necessary definitions. In Section 3, we discuss the general solution of the functional Equation (1). In Section 4, we investigate the stability of Quattuordecic functional Equation (1) in quasi- $\beta$-normed spaces and we provide a counter example to show that the functional Equation (1) is not stable.

\section{Preliminaries}

We recall some basic concepts concerning quasi- $\beta$-normed spaces introduced by J. M. Rassias and H. M. Kim [14] in 2009. Let $\beta$ be a fixed real number with $0<\beta \leq 1$, and let $K$ denote either $R$ or $C$. Let $X$ be linear space over $K$. A quasi- $\beta$-norm $\|$.$\| is a real$ valued function on $X$ satisfying the following three conditions:

1) $\|x\| \geq 0$, for all $x \in X$; and $\|x\|=0$ iff $x=0$,

2) $\|\lambda x\|=|\lambda|^{\beta}\|x\|$ for all $\lambda \in K$, and all $x \in X$,

3) there is a constant $k \geq 1$ such that $\|x+y\| \leq K(\|x\|+\|y\|)$.

For all $x, y \in X$. A quasi- $\beta$-normed space is a pair $(X,\|\|$.$) , where \|$.$\| is a quasi- \beta$ on $X$. The smallest possible $K$ is called the modules of concavity of $\|$.$\| . A quasi- \beta$-Banach space is a complete quasi- $\beta$-normed space. A quasi- $\beta$-norm $\|$.$\| is called a$ $(\beta, p)$-norm $0<\beta \leq 1$ if $\|x+y\|^{p} \leq\|x\|^{p}+\|y\|^{p} \quad \forall x, y \in X$.

In this space a quasi- $\beta$-Banach space is called a $(\beta, p)$-Banach space. We can refer to [18] for the concept of quasi-normed spaces and $p$-Banach spaces. Given a $p$-norm, the formula $d(x, y) \leq\|x-y\|^{p}$ gives us a translation invariant metric on $X$. By the Aoki-Rolewicz theorem, each quasi-norm is equal to some $p$-norm. Since it is much easier to work with $p$-norms then quasi-norms, we restrict our attention mainly to $p$-norms.

Using fixed point theorem, $\mathrm{Xu}$ et al. [18] proved the following impotent lemma.

Lemma 1. Let $i \in-1,1$ be fixed, $a, s \in N$ with $a \geq 2$, and $\varphi: X \rightarrow[0, \infty)$ be a function such that there exists an $L<1$ with $\varphi\left(a^{i} x\right) \leq a^{i s \beta} L \varphi(x)$ for all $x \in X$. Let $f: X \rightarrow Y$ be a mapping satisfying

$$
\left\|f(a x)-a^{s} f(x)\right\|_{Y} \leq \varphi(x) \quad \forall x, y \in X .
$$

Then there exists a uniquely determined mapping $F: X \rightarrow Y$ such that

$$
\|f(x)-F(x)\|_{Y} \leq \frac{1}{a^{s \beta}\left|1-L^{i}\right|} \varphi(x) \quad \forall x \in X .
$$

\section{General Solution of Functional Equation}

In this section, let $X$ and $Y$ be vector spaces. In the following Theorem, we investigate the general solution of the functional Equation (1).

Theorem 1. A function $f: X \rightarrow Y$ is a solution of the Quattuordecic functional Equation (1) if and only if $f$ is of the form $f(x)=A^{14}(x)$ for all $x \in X$, where $A^{14}$ is the diagonal of the 14 -additive symmetric mapping $A_{Q}: X^{14} \rightarrow Y$.

Proof. Assume that $f$ satisfies the functional Equation (1). Replacing $(x, y)$ by $(0,0)$ in (1), we have $f(0)=0$. Replacing $(x, y)$ by $(x, x)$ in (1), we get 


$$
\begin{aligned}
& f(8 x)-14 f(7 x)+91 f(6 x)-364 f(5 x)+1001 f(4 x)-2002 f(3 x) \\
& +3003 f(2 x)-3432 f(x)+3003 f(0)-2002 f(-x)+1001 f(-2 x) \\
& -364 f(-3 x)+91 f(-4 x)-14 f(-5 x)+f(-6 x)=14 ! f(x) .
\end{aligned}
$$

Substituting $(x, y)$ by $(x,-x)$ in $(1)$, we obtain

$$
\begin{aligned}
& f(-6 x)-14 f(-5 x)+91 f(-4 x)-364 f(-3 x)+1001 f(-2 x)-2002 f(-x) \\
& +3003 f(0)-3432 f(x)+3003 f(0)-2002 f(3 x)+1001 f(4 x)-364 f(5 x) \\
& +91 f(6 x)-14 f(7 x)+f(8 x)=14 ! f(-x) .
\end{aligned}
$$

Subtracting Equations (5) and (4), we get

$$
f(-x)=f(x) .
$$

Replacing $(x, y)$ with $(0,2 x)$ in $(1)$, one gets

$$
\begin{aligned}
& 2 f(14 x)-28 f(12 x)+182 f(10 x)-728 f(8 x)+2002 f(6 x)-4004 f(4 x) \\
& +6006 f(2 x)=14 ! f(2 x)
\end{aligned}
$$

and

$$
\begin{aligned}
& f(14 x)-14 f(12 x)+91 f(10 x)-364 f(8 x)+1001 f(6 x)-2002 f(4 x) \\
& +43589142597 f(2 x)=0 .
\end{aligned}
$$

Replacing $(x, y)$ with $(7 x, x)$ in $(1)$, one gets

$$
\begin{aligned}
& f(14 x)-14 f(13 x)+91 f(12 x)-364 f(11 x)+1001 f(10 x)-2002 f(9 x) \\
& +3003 f(8 x)-3432 f(7 x)+3003 f(6 x)-2002 f(5 x)+1001 f(4 x) \\
& -364 f(3 x)+91 f(2 x)=87178291214 f(x) .
\end{aligned}
$$

Subtracting the Equations (7) and (8), we obtain

$$
\begin{aligned}
& 14 f(13 x)-105 f(12 x)+364 f(11 x)-910 f(10 x)+2002 f(9 x) \\
& -3367 f(8 x)+3432 f(7 x)-2002 f(6 x)+2002 f(5 x)-3003 f(4 x) \\
& +364 f(3 x)-43589142688 f(2 x)+87178291214 f(x)=0 .
\end{aligned}
$$

Replacing $(x, y)$ with $(6 x, x)$ in (1) and multiplying by 14 , we have

$$
\begin{aligned}
& 14 f(13 x)-196 f(12 x)+1274 f(11 x)-5096 f(10 x)+14014 f(9 x) \\
& -28028 f(8 x)+42042 f(7 x)-48048 f(6 x)+42042 f(5 x)-28028 f(4 x) \\
& +14014 f(3 x)-5096 f(2 x)-1220496075512 f(x)=0 .
\end{aligned}
$$

Subtracting Equations (9) and (10), we obtain

$$
\begin{aligned}
& 91 f(12 x)+910 f(11 x)+4186 f(10 x)-12012 f(9 x)+24661 f(8 x) \\
& -38610 f(7 x)+46046 f(6 x)-40040 f(5 x)+25025 f(4 x)-13650 f(3 x) \\
& -43589137592 f(2 x)+1307674366726 f(x)=0 .
\end{aligned}
$$

Replacing $(x, y)$ with $(5 x, x)$ in (1) and multiplying by 91 , we have 


$$
\begin{aligned}
& 91 f(12 x)-1274 f(11 x)+8281 f(10 x)-33124 f(9 x)+91091 f(8 x) \\
& -182182 f(7 x)+273273 f(6 x)-312312 f(5 x)+273273 f(4 x) \\
& -182182 f(3 x)+91182 f(2 x)-7933224533598 f(x)=0
\end{aligned}
$$

Subtracting Equations (11) and (12), we have

$$
\begin{aligned}
& 364 f(11 x)-4095 f(10 x)+21112 f(9 x)-66430 f(8 x)+143572 f(7 x) \\
& -227227 f(6 x)+272272 f(5 x)-248248 f(4 x)+168532 f(3 x) \\
& -43589228774 f(2 x)+9240898900324 f(x)=0 .
\end{aligned}
$$

Replacing $(x, y)$ with $(4 x, x)$ in (1) and multiplying by 364 , we have

$$
\begin{aligned}
& 364 f(11 x)-5096 f(10 x)+33124 f(9 x)-132496 f(8 x)+364364 f(7 x) \\
& -728728 f(6 x)+1093092 f(5 x)-1249248 f(4 x)+1093456 f(3 x) \\
& -733824 f(2 x)-317328975599312 f(x)=0
\end{aligned}
$$

Subtracting Equations (13) and (14), we obtain

$$
\begin{aligned}
& 1001 f(10 x)-12012 f(9 x)+66066 f(8 x)-220792 f(7 x) \\
& +501501 f(6 x)-820820 f(5 x)+1001000 f(4 x)-924924 f(3 x) \\
& +43588494950 f(2 x)-40973796499636 f(x)=0 .
\end{aligned}
$$

Replacing $(x, y)$ with $(3 x, x)$ in (1) and multiplying by 1001 , we obtain

$$
\begin{aligned}
& 1001 f(10 x)-14014 f(9 x)+91091 f(8 x)-364364 f(7 x) \\
& +1002001 f(6 x)-2004002 f(5 x))+3007004 f(4 x)-3449446 f(3 x) \\
& +3097094 f(2 x)-87265471859566 f(x)=0
\end{aligned}
$$

Subtracting Equations (15) and (16), one gets

$$
\begin{aligned}
& 2002 f(9 x)-25025 f(8 x)+143572 f(7 x)-500500 f(6 x) \\
& +1183182 f(5 x)-2006004 f(4 x)+2524522 f(3 x) \\
& -43591592044 f(2 x)+128239268359202 f(x)=0 .
\end{aligned}
$$

Replacing $(x, y)$ with $(2 x, x)$ in (1) and multiplying by 2002 , we have

$$
\begin{aligned}
& 2002 f(9 x)-28028 f(8 x)+182182 f(7 x)-728728 f(6 x) \\
& +2006004 f(5 x)-4036032 f(4 x)+6194188 f(3 x) \\
& -7599592 f(2 x)-174530930966392 f(x)=0
\end{aligned}
$$

Subtracting Equations (17) and (18), we obtain

$$
\begin{aligned}
& 3003 f(8 x)-38610 f(7 x)+228228 f(6 x)-822822 f(5 x)+2030028 f(4 x) \\
& -3669666 f(3 x)-43583992452 f(2 x)+302770199325594 f(x)=0 .
\end{aligned}
$$

Replacing $(x, y)$ with $(x, x)$ in (1) and multiply by 3003 , we have

$$
\begin{aligned}
& 3003 f(8 x)-42042 f(7 x)+276276 f(6 x)-1135134 f(5 x)+3279276 f(4 x) \\
& -7105098 f(3 x)+12024012 f(2 x)-261796424791902 f(x)=0 .
\end{aligned}
$$

Subtracting Equations (19) and (20), one gets 


$$
\begin{aligned}
& 3432 f(7 x)-48048 f(6 x)+312312 f(5 x)-1249248 f(4 x) \\
& +3435432 f(3 x))-43596016464 f(2 x)+564566624117496 f(x)=0 .
\end{aligned}
$$

Replacing $(x, y)$ with $(0, x)$ in (1) and multiplying by 1716 , we have

$$
\begin{aligned}
& 3432 f(7 x)-48048 f(6 x)+312312 f(5 x)-1249248 f(4 x) \\
& +3435432 f(3 x)-6870864 f(2 x))+14959793739204 f(x)=0 .
\end{aligned}
$$

Subtracting Equations (20) and (21), we have

$$
-43589145600 f(2 x)+714164561510400 f(x)=0
$$

or

$$
f(2 x)=2^{14} x
$$

On the other hand, one can rewrite the functional Equation (1) in the form

$$
\begin{aligned}
& f(x)-\frac{1}{3432} f(x+7 y)+\frac{7}{1716} f(x+6 y)-\frac{7}{264} f(x+5 y)+\frac{7}{66} f(x+4 y) \\
& -\frac{7}{24} f(x+3 y)+\frac{7}{12} f(x+2 y)-\frac{7}{8} f(x+y)-\frac{7}{8} f(x-y)+\frac{7}{12} f(x-2 y) \\
& -\frac{7}{24} f(x-3 y)+\frac{7}{66} f(x-4 y)-\frac{7}{264} f(x-5 y)+\frac{7}{1716} f(x-6 y) \\
& -\frac{1}{3432} f(x-7 y)+25401600 f(y)=0
\end{aligned}
$$

for all $x \in X$. By ([17], Theorems 3.5 and 3.6), $f$ is a generalized polynomial function of degree at most 14 , that is, $f$ is of the form

$$
\begin{aligned}
f(x)= & A^{14} x+A^{13} x+A^{12} x+A^{11} x+A^{10} x+A^{9} x+A^{8} x+A^{7} x \\
& +A^{6} x+A^{5} x+A^{4} x+A^{3} x+A^{2} x+A^{1} x+A^{0} x
\end{aligned}
$$

for all $x \in X$.

Here, $A^{0} x=A^{0}$ is an arbitrary element of $y$ and $A^{i} x$ is the diagonal of the $i$-additive symmetric map $A_{i}: X^{i} \rightarrow Y \quad(i=1,2,3, \cdots, 14)$ by $f(0)=0$ and $f(-x)=f(x)$, for all $x \in X$, we get $A^{0} x=A^{0}=0$ and the function $f$ is even. Thus, we have

$$
A^{13} x+A^{11} x+A^{9} x+A^{7} x+A^{5} x+A^{3} x+A^{1} x=0
$$

it follows that

$$
f(x)=A^{14} x+A^{12} x+A^{10} x+A^{8} x+A^{6} x+A^{4} x+A^{2} x .
$$

Using Equations (25) and $A^{n}(r x)=r^{n} A^{n}(x)$, we obtain

$$
\begin{aligned}
& 2^{14}\left(A^{14} x+A^{12} x+A^{10} x+A^{8} x+A^{6} x+A^{4} x+A^{2} x\right) \\
& =2^{14} A^{14} x+2^{12} A^{12} x+2^{10} A^{10} x+2^{8} A^{8} x+2^{6} A^{6} x+2^{4} A^{4} x+2^{2} A^{2} x .
\end{aligned}
$$

for all $x \in X$ and $r \in Q$. It follows that

$$
A^{12} x+A^{10} x+A^{8} x+A^{6} x+A^{4} x+A^{2} x=0
$$

for all $x \in X$. Hence $f(x)=A^{14} x$.

Conversely, assume that $f(x)=A^{14} x$ for all $x \in X$, where $A^{14} x$ is the diagonal 
of the 14-additive symmetric map $A_{14}: X^{14} \rightarrow Y$ from

$$
\begin{aligned}
A^{14}(x+y)= & A^{14} x+A^{14} y+14 A^{13.1}(x, y)+91 A^{12.2}(x, y)+364 A^{11.3}(x, y) \\
& +1001 A^{10.4}(x, y)+2002 A^{9.5}(x, y)+3003 A^{8.6}(x, y)+3432 A^{7.7}(x, y) \\
& +3003 A^{6.8}(x, y)+2002 A^{5.9}(x, y)+1001 A^{4.10}(x, y)+364 A^{3.11}(x, y) \\
& +91 A^{2.12}(x, y)+14 A^{1.13}(x, y),
\end{aligned}
$$

and

$$
\begin{aligned}
& A^{14}(r x)=r^{14} A^{14} x, A^{13}(x, r y)=r \cdot A^{13.1}(x, y), A^{12.2}(x, r y)=r^{2} A^{12.2}(x, y), \\
& A^{11.3}(x, r y)=r^{3} A^{11.3}(x, y), A^{10.4}(x, r y)=r^{4} A^{10.4}(x, y), \\
& A^{9.5}(x, r y)=r^{5} A^{9.5}(x, y), A^{8.6}(x, r y)=r^{6} A^{8.6}(x, y), \\
& A^{7.7}(x, r y)=r^{7} A^{7.7}(x, y), A^{6.8}(x, r y)=r^{8} A^{6.8}(x, y), \\
& A^{5.9}(x, r y)=r^{9} A^{5.9}(x, y), A^{4.10}(x, r y)=r^{10} A^{4.10}(x, y), \\
& A^{3.11}(x, r y)=r^{11} A^{3.11}(x, y), A^{2.12}(x, r y)=r^{12} A^{2.12}(x, y), \\
& A^{1.13}(x, r y)=r^{13} A^{1.13}(x, y),
\end{aligned}
$$

for all $x, y \in X$ and $r \in Q$. We see that $f$ satisfies the Equation (1). This completes the proof of the Theorem.

\section{Stability of Quattuordecic Functional Equation}

Throughout this section, we assume that $X$ is a linear space, $Y$ is a $(\beta, p)$ Banach space with $(\beta, p)$-norm \|\|$_{Y}$. Let $K$ be the modulus of concavity of \|\|$_{Y}$. We establish the following stability for the Quarttuordecic functional equation in quasi $\beta$-normed spaces. For a given mapping $f: X \rightarrow Y$, we define the difference operator

$$
\begin{aligned}
D f(x, y)= & f(x+7 y)-14 f(x+6 y)+91 f(x+5 y)-364 f(x+4 y) \\
& +1001 f(x+3 y)-2002 f(x+2 y)+3003 f(x+y)-3432 f(x) \\
& +3003 f(x-y)-2002 f(x-2 y)+1001 f(x-3 y)-364 f(x-4 y) \\
& +91 f(x-5 y)-14 f(x-6 y)+f(x-7 y)-87178291200 f(y) .
\end{aligned}
$$

Theorem 2. Let $i \in\{-1,1\}$ be fixed and $\varphi: X \times X \rightarrow[0, \infty)$ be a function such that there exists an $L<1$ with $\varphi\left(2^{i} x, 2^{i} y\right) \leq 16384^{i \beta} L \varphi(x, y)$ for all $x, y \in X$. Let $f: X \rightarrow Y$ be a mapping satisfying

$$
\|D f(x, y)\|_{Y} \leq \varphi(x, y),
$$

for all $x, y \in X$. Then there exists a unique Quattuordecic mapping $Q: X \rightarrow Y$ such that

$$
\|f(x)-Q(x)\|_{Y} \leq \frac{1}{16384^{\beta}\left\|1-L^{j}\right\|} \varphi_{Q}(x, y),
$$

for all $x, y \in X$, where 


$$
\begin{aligned}
& \varphi_{Q}(x, y)=K \varphi(7 x, x)+\frac{K^{3}}{2^{\beta}} \varphi(0,2 x)+\frac{K^{4}}{50803200} \varphi(0,0)+\frac{K^{11}}{58060800}(\varphi(2 x, 2 x)+\varphi(2 x,-2 x)) \\
& +\frac{K^{12}}{87091200}(\varphi(4 x, 4 x)+\varphi(4 x,-4 x))+\frac{K^{13}}{174182400}(\varphi(6 x, 6 x)+\varphi(6 x,-6 x)) \\
& +\frac{K^{14}}{479001600}(\varphi(8 x, 8 x)+\varphi(8 x,-8 x))+\frac{K^{15}}{1916006400}(\varphi(10 x, 10 x)+\varphi(10 x,-10 x)) \\
& +\frac{K^{16}}{12454041600}(\varphi(12 x, 12 x)+\varphi(12 x,-12 x))+\frac{14^{\beta} K^{8}}{6227020800} \varphi(0,0) \\
& +\frac{K^{17}}{174356582400}(\varphi(14 x, 14 x)+\varphi(14 x,-14 x))+14^{\beta} K^{8} \varphi(6 x, x) \\
& +\frac{14^{\beta} K^{8}}{87178291200}(\varphi(x, x)+\varphi(x,-x))+91^{\beta} K^{7} \varphi(5 x, x)+\frac{91^{\beta} K^{8} \varphi(0,0)}{958003200} \\
& +\frac{91^{\beta} K^{9}}{6227020800}(\varphi(x, x)+\varphi(x,-x))+\frac{91^{\beta} K^{10}}{87178291200}(\varphi(2 x, 2 x)+\varphi(2 x,-2 x)) \\
& +364^{\beta} K^{6} \varphi(4 x, x)+\frac{364^{\beta} K^{7} \varphi(0,0)}{239500800}+\frac{364^{\beta} K^{8}}{958003200}(\varphi(x, x)+\varphi(x,-x)) \\
& +\frac{364^{\beta} K^{9}}{6227020800}(\varphi(2 x, 2 x)+\varphi(2 x,-2 x))+\frac{364^{\beta} K^{10}}{87178291200}(\varphi(3 x, 3 x)+\varphi(3 x,-3 x)) \\
& +1001^{\beta} K^{5} \varphi(3 x, x)+\frac{1001^{\beta} K^{6} \varphi(0,0)}{87091200}+\frac{1001^{\beta} K^{7}}{239500800}(\varphi(x, x)+\varphi(x,-x)) \\
& +\frac{1001^{\beta} K^{8}}{958003200}(\varphi(2 x, 2 x)+\varphi(2 x,-2 x))+\frac{1001^{\beta} K^{9}}{6227020800}(\varphi(3 x, 3 x)+\varphi(3 x,-3 x)) \\
& +\frac{1001^{\beta} K^{10}}{87178291200}(\varphi(4 x, 4 x)+\varphi(4 x,-4 x))+2002^{\beta} K^{4} \varphi(2 x, x)+\frac{2002^{\beta} K^{5} \varphi(0,0)}{43545600} \\
& +\frac{2002^{\beta} K^{6}}{87091200}(\varphi(x, x)+\varphi(x,-x))+\frac{2002^{\beta} K^{7}}{239500800}(\varphi(2 x, 2 x)+\varphi(2 x,-2 x)) \\
& +\frac{2002^{\beta} K^{8}}{958003200}(\varphi(3 x, 3 x)+\varphi(3 x,-3 x))+\frac{2002^{\beta} K^{9}}{6227020800}(\varphi(4 x, 4 x)+\varphi(4 x,-4 x)) \\
& +\frac{2002^{\beta} K^{10}}{87178291200}(\varphi(5 x, 5 x)+\varphi(5 x,-5 x))+3003^{\beta} K^{3} \varphi(x, x)+\frac{3003^{\beta} K^{4} \varphi(0,0)}{29030400} \\
& +\frac{3003^{\beta} K^{5}}{43545600}(\varphi(x, x)+\varphi(x,-x))+\frac{3003^{\beta} K^{6}}{87091200}(\varphi(2 x, 2 x)+\varphi(2 x,-2 x)) \\
& +\frac{3003^{\beta} K^{7}}{239500800}(\varphi(3 x, 3 x)+\varphi(3 x,-3 x))+\frac{3003^{\beta} K^{8}}{958003200}(\varphi(4 x, 4 x)+\varphi(4 x,-4 x)) \\
& +\frac{3003^{\beta} K^{9}}{6227020800}(\varphi(5 x, 5 x)+\varphi(5 x,-5 x))+\frac{3003^{\beta} K^{10}}{87178291200}(\varphi(6 x, 6 x)+\varphi(6 x,-6 x)) \\
& +1716^{\beta} K^{2} \varphi(0, x)+\frac{1716^{\beta} K^{3} \varphi(0,0)}{25401600}+\frac{1716^{\beta} K^{4}}{29030400}(\varphi(x, x)+\varphi(x,-x)) \\
& +\frac{1716^{\beta} K^{5}}{43545600}(\varphi(2 x, 2 x)+\varphi(2 x,-2 x))+\frac{1716^{\beta} K^{6}}{87091200}(\varphi(3 x, 3 x)+\varphi(3 x,-3 x)) \\
& +\frac{1716^{\beta} K^{7}}{239500800}(\varphi(4 x, 4 x)+\varphi(4 x,-4 x))+\frac{1716^{\beta} K^{8}}{958003200}(\varphi(5 x, 5 x)+\varphi(5 x,-5 x)) \\
& +\frac{1716^{\beta} K^{9}}{6227020800}(\varphi(6 x, 6 x)+\varphi(6 x,-6 x))+\frac{1716^{\beta} K^{10}}{87178291200}(\varphi(7 x, 7 x)+\varphi(7 x,-7 x)) \text {. }
\end{aligned}
$$


Proof. Replacing $\quad x=0, y=0$ in (28), we get

$$
\|f(0)\|_{Y} \leq \frac{1}{87178291200} \varphi(0,0) .
$$

Replacing $(x, y)$ by $(x, x)$ in (28), we arrive that

$$
\begin{aligned}
& \| f(8 x)-14 f(7 x)+91 f(6 x)-364 f(5 x)+1001 f(4 x)-2002 f(3 x) \\
& +3003 f(2 x)-3432 f(x)+3003 f(0)-2002 f(-x)+1001 f(-2 x) \\
& -364 f(-3 x)+91 f(-4 x)-14 f(-5 x)+f(-6 x)-87178291200 f(x) \|_{Y} \\
& \leq \varphi(x, x) .
\end{aligned}
$$

Replacing $(x, y)$ by $(x,-x)$ in (28), we have

$$
\begin{aligned}
& \| f(-6 x)-14 f(-5 x)+91 f(-4 x)-364 f(-3 x)+1001 f(-2 x)-2002 f(-x) \\
& +3003 f(0)-3432 f(x)+3003 f(2 x)-2002 f(3 x)+1001 f(4 x)-364 f(5 x) \\
& +91 f(6 x)-14 f(7 x)+f(8 x)-87178291200 f(-x) \|_{Y} \leq \varphi(x,-x) .
\end{aligned}
$$

From Equations (32) and (33), we obtain

$$
\|f(x)-f(-x)\|_{Y} \leq \frac{K}{87178291200}(\varphi(x, x)+\varphi(x,-x)) .
$$

Replacing $(x, y)$ with $(0,2 x)$ in $(28)$, we arrive that

$$
\begin{aligned}
& \| f(14 x)-14 f(12 x)+91 f(10 x)-364 f(8 x)+1001 f(6 x)-2002 f(4 x) \\
& +3003 f(2 x)-3432 f(0)+3003 f(-2 x)-2002 f(-4 x)+1001 f(-6 x) \\
& -364 f(-8 x)+91 f(-10 x)-14 f(-12 x)+f(-14 x)-87178291200 f(2 x) \|_{Y} \\
& \leq \varphi(0,2 x),
\end{aligned}
$$

for all $x \in X$. By (31), (34) and (35), we have

$$
\begin{aligned}
& \|f(14 x)-14 f(12 x)+91 f(10 x)-364 f(8 x)+1001 f(16 x)-2002 f(4 x)-87178285194\|_{Y} \\
& \leq \frac{K}{2^{\beta}} \varphi(0,2 x)+\frac{K^{2}}{50803200} \varphi(0,0)+\frac{K^{3}}{58060800}(\varphi(2 x, 2 x)+\varphi(2 x,-2 x)) \\
& +\frac{K^{4}}{87091200}(\varphi(4 x, 4 x)+\varphi(4 x,-4 x))+\frac{K^{5}}{174182400}(\varphi(6 x, 6 x)+\varphi(6 x,-6 x)) \\
& +\frac{K^{6}}{479001600}(\varphi(8 x, 8 x)+\varphi(8 x,-8 x))+\frac{K^{7}}{1916006400}(\varphi(10 x, 10 x)+\varphi(10 x,-10 x)) \\
& +\frac{K^{8}}{12454041600}(\varphi(12 x, 12 x)+\varphi(12 x,-12 x)) \\
& +\frac{K^{9}}{174356582400}(\varphi(14 x, 14 x)+\varphi(14 x,-14 x)) . \\
& \quad \text { Replacing }(x, y) \text { with }(7 x, x) \text { in }(28), \text { we have } \\
& \| f(14 x)-14 f(13 x)+91 f(12 x)-364 f(11 x)+1001 f(10 x) \\
& \quad-2002 f(9 x)+3003 f(8 x)-3432 f(7 x)+3003 f(6 x)-2002 f(5 x) \\
& +1001 f(4 x)-364 f(3 x)+91 f(2 x)+87178291214 f(x) \|_{Y} \leq K \varphi(7 x, x) .
\end{aligned}
$$

From (36) and (37), we arrive that 


$$
\begin{aligned}
& \| 14 f(13 x)-105 f(12 x)+364 f(11 x)-910 f(10 x)+2002 f(9 x)-3367 f(8 x) \\
&+3432 f(7 x)-2002 f(6 x)+2002 f(5 x)-3003 f(4 x)+364 f(3 x) \\
&-43589142688 f(2 x)+87178291214 f(x) \|_{Y} \\
& \leq K \varphi(7 x, x)+\frac{K^{2}}{2^{\beta}} \varphi(0,2 x)+\frac{K^{3}}{50803200} \varphi(0,0) \\
&+\frac{K^{4}}{58060800}(\varphi(2 x, 2 x)+\varphi(2 x,-2 x)) \\
&+\frac{K^{5}}{87091200}(\varphi(4 x, 4 x)+\varphi(4 x,-4 x)) \\
&+\frac{K^{6}}{174182400}(\varphi(6 x, 6 x)+\varphi(6 x,-6 x)) \\
&+\frac{K^{7}}{479001600}(\varphi(8 x, 8 x)+\varphi(8 x,-8 x)) \\
&+\frac{K^{8}}{1916006400}(\varphi(10 x, 10 x)+\varphi(10 x,-10 x)) \\
&+\frac{K^{9}}{12454041600}(\varphi(12 x, 12 x)+\varphi(12 x,-12 x)) \\
&+\frac{K^{10}}{174356582400}(\varphi(14 x, 14 x)+\varphi(14 x,-14 x))
\end{aligned}
$$

Replacing $(x, y)$ with $(6 x, x)$ in (28), one finds that

$$
\begin{aligned}
& \| 14 f(13 x)-196 f(12 x)+1274 f(11 x)-5096 f(10 x)+14014 f((9 x) \\
& -28028 f(8 x)+42042 f(7 x)-48048 f(6 x)+42042 f(5 x)-28028 f(4 x) \\
& +14014 f(3 x)-5096 f(2 x)-1220496075512 f(x) \|_{Y} \\
& \leq 14^{\beta} K \varphi(6 x, x)+\frac{14^{\beta} K^{2}}{6227020800} \varphi(0,0)+\frac{14^{\beta} K^{3}}{87178291200}(\varphi(x, x)+\varphi(x,-x)) .
\end{aligned}
$$

Utilizing (38) and (39), we find that

$$
\begin{aligned}
\| & 91 f(12 x)+910 f(11 x)+4186 f(10 x)-12012 f(19 x)+24661 f(8 x) \\
- & 38610 f(7 x)+46046 f(6 x)-40040 f(5 x)+25025 f(4 x)-13650 f(3 x) \\
- & 43589137592 f(2 x)+1307674366726 f(x) \|_{Y} \\
\leq & K^{2} \varphi(7 x, x)+\frac{K^{3}}{2^{\beta}} \varphi(0,2 x)+\frac{K^{4}}{50803200} \varphi(0,0)+\frac{K^{5}}{58060800}(\varphi(2 x, 2 x)+\varphi(2 x,-2 x)) \\
& +\frac{K^{6}}{87091200}(\varphi(4 x, 4 x)+\varphi(4 x,-4 x))+\frac{K^{7}}{174182400}(\varphi(6 x, 6 x)+\varphi(6 x,-6 x)) \\
& +\frac{K^{8}}{479001600}(\varphi(8 x, 8 x)+\varphi(8 x,-8 x))+\frac{K^{9}}{1916006400}(\varphi(10 x, 10 x)+\varphi(10 x,-10 x)) \\
& +\frac{K^{10}}{12454041600}(\varphi(12 x, 12 x)+\varphi(12 x,-12 x))+14^{\beta} K^{2} \varphi(6 x, x)+\frac{14^{\beta} K^{2}}{6227020800} \varphi(0,0) \\
& +\frac{K^{11}}{174356582400}(\varphi(14 x, 14 x)+\varphi(14 x,-14 x))+\frac{14^{\beta} K^{2}}{87178291200}(\varphi(x, x)+\varphi(x,-x)) .
\end{aligned}
$$

Replacing $(x, y)$ with $(5 x, x)$ in $(28)$, we obtain 


$$
\begin{aligned}
& \| 91 f(12 x)-1274 f(11 x)+8281 f(10 x)-33124 f(9 x)+91091 f(8 x) \\
& -182182 f(7 x)+273273 f(6 x)+312312 f(5 x)+273273 f(4 x) \\
& -182182 f(3 x)+91182 f(2 x)-7933224533598 f(x) \|_{Y} \\
& \leq 91^{\beta} K \varphi(5 x, x)+\frac{91^{\beta} K^{2} \varphi(0,0)}{958003200}+\frac{91^{\beta} K^{3}}{6227020800}(\varphi(x, x)+\varphi(x,-x)) \\
& +\frac{91^{\beta} K^{4}}{87178291200}(\varphi(2 x, 2 x)+\varphi(2 x,-2 x)) .
\end{aligned}
$$

From (40) and (41), we arrive at

$$
\begin{aligned}
& \| 364 f(11 x)-4095 f(10 x)+21112 f(9 x)-66430 f(8 x)+143572 f(7 x) \\
&- 227227 f(6 x)+272272 f(5 x)-248248 f(4 x)+168532 f(3 x) \\
&- 43589228774 f(2 x)+9240898900324 f(x) \|_{Y} \\
& \leq K^{3} \varphi(7 x, x)+\frac{K^{3}}{2^{\beta}} \varphi(0,2 x)+\frac{K^{4}}{50803200} \varphi(0,0) \\
&+\frac{K^{6}}{58060800}(\varphi(2 x, 2 x)+\varphi(2 x,-2 x)) \\
&+\frac{K^{7}}{87091200}(\varphi(4 x, 4 x)+\varphi(4 x,-4 x))+\frac{K^{8}}{174182400}(\varphi(6 x, 6 x)+\varphi(6 x,-6 x)) \\
&+\frac{K^{9}}{479001600}(\varphi(8 x, 8 x)+\varphi(8 x,-8 x)) \\
&+\frac{K^{10}}{1916006400}(\varphi(10 x, 10 x)+\varphi(10 x,-10 x)) \\
&+\frac{K^{11}}{12454041600}(\varphi(12 x, 12 x)+\varphi(12 x,-12 x))+14^{\beta} K^{3} \varphi(6 x, x) \\
&+\frac{K^{12}}{174356582400}(\varphi(14 x, 14 x)+\varphi(14 x,-14 x))+\frac{14^{\beta} K^{3}}{6227020800} \varphi(0,0) \\
&+\frac{14^{\beta} K^{3}}{87178291200}(\varphi(x, x)+\varphi(x,-x))+91^{\beta} K^{2} \varphi(5 x, x)+\frac{91^{\beta} K^{3} \varphi(0,0)}{958003200} \\
&+\frac{91^{\beta} K^{4}}{6227020800}(\varphi(x, x)+\varphi(x,-x))+\frac{91^{\beta} K^{5}}{87178291200}(\varphi(2 x, 2 x)+\varphi(2 x,-2 x)) .
\end{aligned}
$$

Replacing $(x, y)$ with $(4 x, x)$ in $(28)$, we obtain

$$
\begin{aligned}
& \| 364 f(11 x)-5096 f(10 x)+33124 f(9 x)-132496 f(8 x)+364364 f(7 x) \\
& -728728 f(6 x)+1093092 f(5 x)-1249248 f(4 x)+1093456 f(3 x) \\
& -733824 f(2 x)-317328975599312 f(x) \|_{Y} \\
& \leq 364^{\beta} K \varphi(4 x, x)+\frac{364^{\beta} K^{2} \varphi(0,0)}{239500800}+\frac{364^{\beta} K^{3}}{958003200}(\varphi(x, x)+\varphi(x,-x)) \\
& +\frac{364^{\beta} K^{4}}{6227020800}(\varphi(2 x, 2 x)+\varphi(2 x,-2 x)) \\
& +\frac{364^{\beta} K^{5}}{87178291200}(\varphi(3 x, 3 x)+\varphi(3 x,-3 x)) .
\end{aligned}
$$

Using Equations (42) and (43), we get 


$$
\begin{aligned}
& \| 1001 f(10 x)-12012 f(9 x)+66066 f(8 x)-220792 f(7 x)+501501 f(6 x) \\
& -820820 f(5 x)+1001000 f(4 x)-924924 f(3 x)+43588494950 f(2 x) \\
& -40973796499636 f(x) \|_{Y} \\
& \leq K \varphi(7 x, x)+\frac{K^{3}}{2^{\beta}} \varphi(0,2 x)+\frac{K^{4}}{50803200} \varphi(0,0) \\
& +\frac{K^{7}}{58060800}(\varphi(2 x, 2 x)+\varphi(2 x,-2 x)) \\
& +\frac{K^{8}}{87091200}(\varphi(4 x, 4 x)+\varphi(4 x,-4 x))+\frac{K^{9}}{174182400}(\varphi(6 x, 6 x)+\varphi(6 x,-6 x)) \\
& +\frac{K^{10}}{479001600}(\varphi(8 x, 8 x)+\varphi(8 x,-8 x)) \\
& +\frac{K^{11}}{1916006400}(\varphi(10 x, 10 x)+\varphi(10 x,-10 x)) \\
& +\frac{K^{12}}{12454041600}(\varphi(12 x, 12 x)+\varphi(12 x,-12 x))+14^{\beta} K^{4} \varphi(6 x, x) \\
& +\frac{K^{13}}{174356582400}(\varphi(14 x, 14 x)+\varphi(14 x,-14 x))+\frac{14^{\beta} K^{4}}{6227020800} \varphi(0,0) \\
& +\frac{14^{\beta} K^{4}}{87178291200}(\varphi(x, x)+\varphi(x,-x))+91^{\beta} K^{3} \varphi(5 x, x)+\frac{91^{\beta} K^{4} \varphi(0,0)}{958003200} \\
& +\frac{91^{\beta} K^{5}}{6227020800}(\varphi(x, x)+\varphi(x,-x))+\frac{91^{\beta} K^{6}}{87178291200}(\varphi(2 x, 2 x)+\varphi(2 x,-2 x)) \\
& +364^{\beta} K^{2} \varphi(4 x, x)+\frac{364^{\beta} K^{3} \varphi(0,0)}{239500800}+\frac{364^{\beta} K^{4}}{958003200}(\varphi(x, x)+\varphi(x,-x)) \\
& +\frac{364^{\beta} K^{5}}{6227020800}(\varphi(2 x, 2 x)+\varphi(2 x,-2 x)) \\
& +\frac{364^{\beta} K^{6}}{87178291200}(\varphi(3 x, 3 x)+\varphi(3 x,-3 x)) \text {. }
\end{aligned}
$$

Replacing $(x, y)$ with $(3 x, x)$ in (28), one finds that

$$
\begin{aligned}
\| & 1001 f(10 x)-14014 f(9 x)+91091 f(8 x)-364364 f(7 x) \\
+ & 1002001 f(6 x)-2004002 f(5 x)+3007004 f(4 x)-3449446 f(3 x) \\
+ & 3097094 f(2 x)-87265471859566 f(x) \|_{Y} \\
\leq & 1001^{\beta} K \varphi(3 x, x)+\frac{1001^{\beta} K^{2} \varphi(0,0)}{87091200}+\frac{1001^{\beta} K^{3}}{239500800}(\varphi(x, x)+\varphi(x,-x)) \\
& +\frac{1001^{\beta} K^{4}}{958003200}(\varphi(2 x, 2 x)+\varphi(2 x,-2 x)) \\
& +\frac{1001^{\beta} K^{5}}{6227020800}(\varphi(3 x, 3 x)+\varphi(3 x,-3 x)) \\
& +\frac{1001^{\beta} K^{6}}{87178291200}(\varphi(4 x, 4 x)+\varphi(4 x,-4 x)) .
\end{aligned}
$$

From (44) and (45), we arrive at 


$$
\begin{aligned}
& \text { ||2002 } f(9 x)-25025 f(8 x)+143572 f(7 x)-500500 f(6 x) \\
& +1183182 f(5 x)-2006004 f(4 x)+2524522 f(3 x) \\
& -43591592044 f(2 x)+128239268359202 f(x) \|_{Y} \\
& \leq K^{5} \varphi(7 x, x)+\frac{K^{3}}{2^{\beta}} \varphi(0,2 x)+\frac{K^{4}}{50803200} \varphi(0,0) \\
& +\frac{K^{8}}{58060800}(\varphi(2 x, 2 x)+\varphi(2 x,-2 x)) \\
& +\frac{K^{9}}{87091200}(\varphi(4 x, 4 x)+\varphi(4 x,-4 x))+\frac{K^{10}}{174182400}(\varphi(6 x, 6 x)+\varphi(6 x,-6 x)) \\
& +\frac{K^{11}}{479001600}(\varphi(8 x, 8 x)+\varphi(8 x,-8 x)) \\
& +\frac{K^{12}}{1916006400}(\varphi(10 x, 10 x)+\varphi(10 x,-10 x)) \\
& +\frac{K^{13}}{12454041600}(\varphi(12 x, 12 x)+\varphi(12 x,-12 x))+14^{\beta} K^{5} \varphi(6 x, x) \\
& +\frac{K^{14}}{174356582400}(\varphi(14 x, 14 x)+\varphi(14 x,-14 x))+\frac{14^{\beta} K^{5}}{6227020800} \varphi(0,0) \\
& +\frac{14^{\beta} K^{5}}{87178291200}(\varphi(x, x)+\varphi(x,-x))+91^{\beta} K^{4} \varphi(5 x, x)+\frac{91^{\beta} K^{5} \varphi(0,0)}{958003200} \\
& +\frac{91^{\beta} K^{6}}{6227020800}(\varphi(x, x)+\varphi(x,-x))+\frac{91^{\beta} K^{7}}{87178291200}(\varphi(2 x, 2 x)+\varphi(2 x,-2 x)) \\
& +364^{\beta} K^{3} \varphi(4 x, x)+\frac{364^{\beta} K^{4} \varphi(0,0)}{239500800}+\frac{364^{\beta} K^{5}}{958003200}(\varphi(x, x)+\varphi(x,-x)) \\
& +\frac{364^{\beta} K^{6}}{6227020800}(\varphi(2 x, 2 x)+\varphi(2 x,-2 x))+\frac{364^{\beta} K^{7}}{87178291200}(\varphi(3 x, 3 x)+\varphi(3 x,-3 x)) \\
& +1001^{\beta} K^{2} \varphi(3 x, x)+\frac{1001^{\beta} K^{3} \varphi(0,0)}{87091200}+\frac{1001^{\beta} K^{4}}{239500800}(\varphi(x, x)+\varphi(x,-x)) \\
& +\frac{1001^{\beta} K^{5}}{958003200}(\varphi(2 x, 2 x)+\varphi(2 x,-2 x))+\frac{1001^{\beta} K^{6}}{6227020800}(\varphi(3 x, 3 x)+\varphi(3 x,-3 x)) \\
& +\frac{1001^{\beta} K^{7}}{87178291200}(\varphi(4 x, 4 x)+\varphi(4 x,-4 x)) \text {. }
\end{aligned}
$$

Replacing $(x, y)$ with $(2 x, x)$ in $(28)$, we obtain

$$
\begin{aligned}
\| & 2002 f(9 x)-28028 f(8 x)+182182 f(7 x)-728728 f(6 x)+2006004 f(5 x) \\
- & 4036032 f(4 x)+6194188 f(3 x)-7599592 f(2 x)-174530930966392 f(x) \|_{Y} \\
\leq & 2002^{\beta} K^{1} \varphi(2 x, x)+\frac{2002^{\beta} K^{2} \varphi(0,0)}{43545600}+\frac{2002^{\beta} K^{3}}{87091200}(\varphi(x, x)+\varphi(x,-x)) \\
& +\frac{2002^{\beta} K^{4}}{239500800}(\varphi(2 x, 2 x)+\varphi(2 x,-2 x))+\frac{2002^{\beta} K^{5}}{958003200}(\varphi(3 x, 3 x)+\varphi(3 x,-3 x)) \\
& +\frac{2002^{\beta} K^{6}}{6227020800}(\varphi(4 x, 4 x)+\varphi(4 x,-4 x)) \\
& +\frac{2002^{\beta} K^{7}}{87178291200}(\varphi(5 x, 5 x)+\varphi(5 x,-5 x)) .
\end{aligned}
$$

Using Equations (46) and (47), one gets that 


$$
\begin{aligned}
& \mid 3003 f(8 x)-38610 f(7 x)+228228 f(6 x)-822822 f(5 x)+2030028 f(4 x) \\
& -3669666 f(3 x)-43583992452 f(2 x)+302770199325594 f(x) \|_{Y} \\
& \leq K^{6} \varphi(7 x, x)+\frac{K^{3}}{2^{\beta}} \varphi(0,2 x)+\frac{K^{4}}{50803200} \varphi(0,0)+\frac{K^{9}}{58060800}(\varphi(2 x, 2 x)+\varphi(2 x,-2 x)) \\
& +\frac{K^{10}}{87091200}(\varphi(4 x, 4 x)+\varphi(4 x,-4 x))+\frac{K^{11}}{174182400}(\varphi(6 x, 6 x)+\varphi(6 x,-6 x)) \\
& +\frac{K^{12}}{479001600}(\varphi(8 x, 8 x)+\varphi(8 x,-8 x))+\frac{K^{13}}{1916006400}(\varphi(10 x, 10 x)+\varphi(10 x,-10 x)) \\
& +\frac{K^{14}}{12454041600}(\varphi(12 x, 12 x)+\varphi(12 x,-12 x))+14^{\beta} K^{6} \varphi(6 x, x) \\
& +\frac{K^{15}}{174356582400}(\varphi(14 x, 14 x)+\varphi(14 x,-14 x))+\frac{14^{\beta} K^{6}}{6227020800} \varphi(0,0) \\
& +\frac{14^{\beta} K^{6}}{87178291200}(\varphi(x, x)+\varphi(x,-x))+91^{\beta} K^{5} \varphi(5 x, x)+\frac{91^{\beta} K^{6} \varphi(0,0)}{958003200} \\
& +\frac{91^{\beta} K^{7}}{6227020800}(\varphi(x, x)+\varphi(x,-x))+\frac{91^{\beta} K^{8}}{87178291200}(\varphi(2 x, 2 x)+\varphi(2 x,-2 x)) \\
& +364^{\beta} K^{4} \varphi(4 x, x)+\frac{364^{\beta} K^{5} \varphi(0,0)}{239500800}+\frac{364^{\beta} K^{6}}{958003200}(\varphi(x, x)+\varphi(x,-x)) \\
& +\frac{364^{\beta} K^{7}}{6227020800}(\varphi(2 x, 2 x)+\varphi(2 x,-2 x))+\frac{364^{\beta} K^{8}}{87178291200}(\varphi(3 x, 3 x)+\varphi(3 x,-3 x)) \\
& +1001^{\beta} K^{3} \varphi(3 x, x)+\frac{1001^{\beta} K^{4} \varphi(0,0)}{87091200}+\frac{1001^{\beta} K^{5}}{239500800}(\varphi(x, x)+\varphi(x,-x)) \\
& +\frac{1001^{\beta} K^{6}}{958003200}(\varphi(2 x, 2 x)+\varphi(2 x,-2 x))+\frac{1001^{\beta} K^{7}}{6227020800}(\varphi(3 x, 3 x)+\varphi(3 x,-3 x)) \\
& +\frac{1001^{\beta} K^{8}}{87178291200}(\varphi(4 x, 4 x)+\varphi(4 x,-4 x))+2002^{\beta} K^{2} \varphi(2 x, x)+\frac{2002^{\beta} K^{3} \varphi(0,0)}{43545600} \\
& +\frac{2002^{\beta} K^{4}}{87091200}(\varphi(x, x)+\varphi(x,-x))+\frac{2002^{\beta} K^{5}}{239500800}(\varphi(2 x, 2 x)+\varphi(2 x,-2 x)) \\
& +\frac{2002^{\beta} K^{6}}{958003200}(\varphi(3 x, 3 x)+\varphi(3 x,-3 x))+\frac{2002^{\beta} K^{7}}{6227020800}(\varphi(4 x, 4 x)+\varphi(4 x,-4 x)) \\
& +\frac{2002^{\beta} K^{8}}{87178291200}(\varphi(5 x, 5 x)+\varphi(5 x,-5 x)) \text {. } \\
& \text { Replacing }(x, y) \text { with }(x, x) \text { in (28), we have } \\
& \text { ||3003 } f(8 x)-42042 f(7 x)+276276 f(6 x)-1135134 f(5 x) \\
& +3279276 f(4 x)-7105098 f(3 x)+12024012 f(2 x)-261796424791902 f(x) \|_{Y} \\
& \leq 3003^{\beta} K^{1} \varphi(x, x)+\frac{3003^{\beta} K^{2} \varphi(0,0)}{29030400}+\frac{3003^{\beta} K^{3}}{43545600}(\varphi(x, x)+\varphi(x,-x)) \\
& +\frac{3003^{\beta} K^{4}}{87091200}(\varphi(2 x, 2 x)+\varphi(2 x,-2 x))+\frac{3003^{\beta} K^{5}}{239500800}(\varphi(3 x, 3 x)+\varphi(3 x,-3 x)) \\
& +\frac{3003^{\beta} K^{6}}{958003200}(\varphi(4 x, 4 x)+\varphi(4 x,-4 x))+\frac{3003^{\beta} K^{7}}{6227020800}(\varphi(5 x, 5 x)+\varphi(5 x,-5 x)) \\
& +\frac{3003^{\beta} K^{8}}{87178291200}(\varphi(6 x, 6 x)+\varphi(6 x,-6 x)) \text {. }
\end{aligned}
$$


Using Equation (48) and (49), we obtain

||3432 $f(7 x)-48048 f(6 x)+312312 f(5 x)-1249248 f(4 x)$

$+3435432 f(3 x))-43596016464 f(2 x)+564566624117496 f(x) \|_{Y}$

$\leq K^{7} \varphi(7 x, x)+\frac{K^{3}}{2^{\beta}} \varphi(0,2 x)+\frac{K^{4}}{50803200} \varphi(0,0)+\frac{K^{10}}{58060800}(\varphi(2 x, 2 x)+\varphi(2 x,-2 x))$

$+\frac{K^{11}}{87091200}(\varphi(4 x, 4 x)+\varphi(4 x,-4 x))+\frac{K^{12}}{174182400}(\varphi(6 x, 6 x)+\varphi(6 x,-6 x))$

$+\frac{K^{13}}{479001600}(\varphi(8 x, 8 x)+\varphi(8 x,-8 x))+\frac{K^{14}}{1916006400}(\varphi(10 x, 10 x)+\varphi(10 x,-10 x))$

$+\frac{K^{15}}{12454041600}(\varphi(12 x, 12 x)+\varphi(12 x,-12 x))+14^{\beta} K^{7} \varphi(6 x, x)$

$+\frac{K^{16}}{174356582400}(\varphi(14 x, 14 x)+\varphi(14 x,-14 x))+\frac{14^{\beta} K^{7}}{6227020800} \varphi(0,0)$

$+\frac{14^{\beta} K^{7}}{87178291200}(\varphi(x, x)+\varphi(x,-x))+91^{\beta} K^{6} \varphi(5 x, x)+\frac{91^{\beta} K^{7} \varphi(0,0)}{958003200}$

$+\frac{91^{\beta} K^{8}}{6227020800}(\varphi(x, x)+\varphi(x,-x))+\frac{91^{\beta} K^{9}}{87178291200}(\varphi(2 x, 2 x)+\varphi(2 x,-2 x))$

$+364^{\beta} K^{5} \varphi(4 x, x)+\frac{364^{\beta} K^{6} \varphi(0,0)}{239500800}+\frac{364^{\beta} K^{7}}{958003200}(\varphi(x, x)+\varphi(x,-x))$

$+\frac{364^{\beta} K^{8}}{6227020800}(\varphi(2 x, 2 x)+\varphi(2 x,-2 x))+\frac{364^{\beta} K^{9}}{87178291200}(\varphi(3 x, 3 x)+\varphi(3 x,-3 x))$

$+1001^{\beta} K^{4} \varphi(3 x, x)+\frac{1001^{\beta} K^{5} \varphi(0,0)}{87091200}+\frac{1001^{\beta} K^{6}}{239500800}(\varphi(x, x)+\varphi(x,-x))$

$+\frac{1001^{\beta} K^{7}}{958003200}(\varphi(2 x, 2 x)+\varphi(2 x,-2 x))+\frac{1001^{\beta} K^{8}}{6227020800}(\varphi(3 x, 3 x)+\varphi(3 x,-3 x))$

$+\frac{1001^{\beta} K^{9}}{87178291200}(\varphi(4 x, 4 x)+\varphi(4 x,-4 x))+2002^{\beta} K^{3} \varphi(2 x, x)+\frac{2002^{\beta} K^{4} \varphi(0,0)}{43545600}$

$+\frac{2002^{\beta} K^{5}}{87091200}(\varphi(x, x)+\varphi(x,-x))+\frac{2002^{\beta} K^{6}}{239500800}(\varphi(2 x, 2 x)+\varphi(2 x,-2 x))$

$+\frac{2002^{\beta} K^{7}}{958003200}(\varphi(3 x, 3 x)+\varphi(3 x,-3 x))+\frac{2002^{\beta} K^{8}}{6227020800}(\varphi(4 x, 4 x)+\varphi(4 x,-4 x))$

$+\frac{2002^{\beta} K^{9}}{87178291200}(\varphi(5 x, 5 x)+\varphi(5 x,-5 x))+3003^{\beta} K^{2} \varphi(x, x)+\frac{3003^{\beta} K^{3} \varphi(0,0)}{29030400}$

$+\frac{3003^{\beta} K^{4}}{43545600}(\varphi(x, x)+\varphi(x,-x))+\frac{3003^{\beta} K^{5}}{87091200}(\varphi(2 x, 2 x)+\varphi(2 x,-2 x))$

$+\frac{3003^{\beta} K^{6}}{239500800}(\varphi(3 x, 3 x)+\varphi(3 x,-3 x))+\frac{3003^{\beta} K^{7}}{958003200}(\varphi(4 x, 4 x)+\varphi(4 x,-4 x))$

$+\frac{3003^{\beta} K^{8}}{6227020800}(\varphi(5 x, 5 x)+\varphi(5 x,-5 x))+\frac{3003^{\beta} K^{9}}{87178291200}(\varphi(6 x, 6 x)+\varphi(6 x,-6 x))$.

Replacing $(x, y)$ with $(0, x)$ in $(28)$, we obtain 


$$
\begin{aligned}
\| & 3432 f(7 x)-48048 f(6 x)+312312 f(5 x)-1249248 f(4 x) \\
+ & 3435432 f(3 x)-6870864 f(2 x)+14959793739204 f(x) \|_{Y} \\
\leq & 1716^{\beta} K^{1} \varphi(0, x)+\frac{1716^{\beta} K^{2} \varphi(0,0)}{25401600}+\frac{1716^{\beta} K^{3}}{29030400}(\varphi(x, x)+\varphi(x,-x)) \\
& +\frac{1716^{\beta} K^{4}}{43545600}(\varphi(2 x, 2 x)+\varphi(2 x,-2 x))+\frac{1716^{\beta} K^{5}}{87091200}(\varphi(3 x, 3 x)+\varphi(3 x,-3 x)) \\
& +\frac{1716^{\beta} K^{6}}{239500800}(\varphi(4 x, 4 x)+\varphi(4 x,-4 x))+\frac{1716^{\beta} K^{7}}{958003200}(\varphi(5 x, 5 x)+\varphi(5 x,-5 x)) \\
& +\frac{1716^{\beta} K^{8}}{6227020800}(\varphi(6 x, 6 x)+\varphi(6 x,-6 x))+\frac{1716^{\beta} K^{9}}{87178291200}(\varphi(7 x, 7 x)+\varphi(7 x,-7 x)) .
\end{aligned}
$$

From (50) and (51), we arrive at

$$
\begin{aligned}
& \|-43589145600 f(2 x)+714164561510400 f(x)\|_{Y}=K^{8} \varphi(7 x, x)+\frac{K^{3}}{2^{\beta}} \varphi(0,2 x)+\frac{K^{4}}{50803200} \varphi(0,0) \\
& +\frac{K^{11}}{58060800}(\varphi(2 x, 2 x)+\varphi(2 x,-2 x))+\frac{K^{12}}{87091200}(\varphi(4 x, 4 x)+\varphi(4 x,-4 x))+\frac{K^{13}}{174182400}(\varphi(6 x, 6 x)+\varphi(6 x,-6 x)) \\
& +\frac{K^{14}}{479001600}(\varphi(8 x, 8 x)+\varphi(8 x,-8 x))+\frac{K^{15}}{1916006400}(\varphi(10 x, 10 x)+\varphi(10 x,-10 x)) \\
& +\frac{K^{16}}{12454041600}(\varphi(12 x, 12 x)+\varphi(12 x,-12 x))+\frac{14^{\beta} K^{8}}{6227020800} \varphi(0,0)+\frac{K^{17}}{174356582400}(\varphi(14 x, 14 x)+\varphi(14 x,-14 x)) \\
& +14^{\beta} K^{8} \varphi(6 x, x)+\frac{14^{\beta} K^{8}}{87178291200}(\varphi(x, x)+\varphi(x,-x))+91^{\beta} K^{7} \varphi(5 x, x)+\frac{91^{\beta} K^{8} \varphi(0,0)}{958003200}+\frac{91^{\beta} K^{9}}{6227020800}(\varphi(x, x)+\varphi(x,-x)) \\
& +\frac{91^{\beta} K^{10}}{87178291200}(\varphi(2 x, 2 x)+\varphi(2 x,-2 x))+364^{\beta} K^{6} \varphi(4 x, x)+\frac{364^{\beta} K^{7} \varphi(0,0)}{239500800}+\frac{364^{\beta} K^{8}}{958003200}(\varphi(x, x)+\varphi(x,-x)) \\
& +\frac{364^{\beta} K^{9}}{6227020800}(\varphi(2 x, 2 x)+\varphi(2 x,-2 x))+\frac{364^{\beta} K^{10}}{87178291200}(\varphi(3 x, 3 x)+\varphi(3 x,-3 x))+1001^{\beta} K^{5} \varphi(3 x, x)+\frac{1001^{\beta} K^{6} \varphi(0,0)}{87091200} \\
& +\frac{1001^{\beta} K^{7}}{239500800}(\varphi(x, x)+\varphi(x,-x))+\frac{1001^{\beta} K^{8}}{958003200}(\varphi(2 x, 2 x)+\varphi(2 x,-2 x))+\frac{1001^{\beta} K^{9}}{6227020800}(\varphi(3 x, 3 x)+\varphi(3 x,-3 x)) \\
& +\frac{1001^{\beta} K^{10}}{87178291200}(\varphi(4 x, 4 x)+\varphi(4 x,-4 x))+2002^{\beta} K^{4} \varphi(2 x, x)+\frac{2002^{\beta} K^{5} \varphi(0,0)}{43545600}+\frac{2002^{\beta} K^{6}}{87091200}(\varphi(x, x)+\varphi(x,-x)) \\
& +\frac{2002^{\beta} K^{7}}{239500800}(\varphi(2 x, 2 x)+\varphi(2 x,-2 x))+\frac{2002^{\beta} K^{8}}{958003200}(\varphi(3 x, 3 x)+\varphi(3 x,-3 x))+\frac{2002^{\beta} K^{9}}{6227020800}(\varphi(4 x, 4 x)+\varphi(4 x,-4 x)) \\
& +\frac{2002^{\beta} K^{10}}{87178291200}(\varphi(5 x, 5 x)+\varphi(5 x,-5 x))+3003^{\beta} K^{3} \varphi(x, x)+\frac{3003^{\beta} K^{4} \varphi(0,0)}{29030400}+\frac{3003^{\beta} K^{5}}{43545600}(\varphi(x, x)+\varphi(x,-x)) \\
& +\frac{3003^{\beta} K^{6}}{87091200}(\varphi(2 x, 2 x)+\varphi(2 x,-2 x))+\frac{3003^{\beta} K^{7}}{239500800}(\varphi(3 x, 3 x)+\varphi(3 x,-3 x))+\frac{3003^{\beta} K^{8}}{958003200}(\varphi(4 x, 4 x)+\varphi(4 x,-4 x)) \\
& +\frac{3003^{\beta} K^{9}}{6227020800}(\varphi(5 x, 5 x)+\varphi(5 x,-5 x))+\frac{3003^{\beta} K^{10}}{87178291200}(\varphi(6 x, 6 x)+\varphi(6 x,-6 x))+1716^{\beta} K^{2} \varphi(0, x)+\frac{1716^{\beta} K^{3} \varphi(0,0)}{25401600} \\
& +\frac{1716^{\beta} K^{4}}{29030400}(\varphi(x, x)+\varphi(x,-x))+\frac{1716^{\beta} K^{5}}{43545600}(\varphi(2 x, 2 x)+\varphi(2 x,-2 x))+\frac{1716^{\beta} K^{6}}{87091200}(\varphi(3 x, 3 x)+\varphi(3 x,-3 x)) \\
& +\frac{1716^{\beta} K^{7}}{239500800}(\varphi(4 x, 4 x)+\varphi(4 x,-4 x))+\frac{1716^{\beta} K^{8}}{958003200}(\varphi(5 x, 5 x)+\varphi(5 x,-5 x))+\frac{1716^{\beta} K^{9}}{6227020800}(\varphi(6 x, 6 x)+\varphi(6 x,-6 x)) \\
& +\frac{1716^{\beta} K^{10}}{87178291200}(\varphi(7 x, 7 x)+\varphi(7 x,-7 x)) \text {. }
\end{aligned}
$$


Therefore,

$$
\left\|f(2 x)-2^{14} f(x)\right\| \leq \varphi_{14}(x)
$$

for all $x \in X$. By Lemma 2.1, there exists a unique mapping $Q: X \rightarrow Y$ such that

$$
Q(2 x)=2^{14} Q(x)
$$

and

$$
\|f(x)-Q(x)\|_{y} \leq \frac{1}{16384^{\beta}\left|1-L^{i}\right|} \varphi_{14}(x)
$$

for all $x \in X$. It remains to show that $\mathrm{Q}$ is a Quattuordecic mapping. From (28), we have

$$
\begin{aligned}
\left\|\frac{D f\left(2^{i m}(x), 2^{i m}(y)\right)}{16384^{i m}}\right\|_{y} & \leq 16384^{i m \beta} \varphi\left(2^{i m}(x), 2^{i m}(y)\right) \\
& \leq 16384^{i m \beta}\left(16384^{i \beta} L\right)^{m} \varphi(x, y) \\
& =L^{m} \varphi(x, y)
\end{aligned}
$$

for all $x, y \in X$ and $m \in N$. Here $\|D Q(x, y)\|_{Y}=0$, for all $x, y \in X$.

Therefore, the mapping $Q: X \rightarrow Y$ is a Quattuordecic mapping. The following corollary is an immediate consequence of Theorem 4.1 concerning the stability of Quattuordecic functional Equation (1).

Corollary 1. Let $X$ be a quasi $\alpha$-normed space with quasi $\alpha$-norm $\|\cdot\|_{X}$, and let $Y$ be a $(\beta, p)$ Banach Space with $(\beta, p)$-norm \|\|$_{Y}$. Let $\delta, \lambda$ be a positive number with $\lambda \neq \frac{14 \beta}{\alpha}$ and let $f: X \rightarrow Y$ be a mapping satisfying

$$
\|D f(x, y)\|_{Y} \leq \delta\left(\|x\|_{X}^{Y}+\|y\|_{X}^{Y}\right)
$$

for all $x, y \in X$. Then there exists a unique quattuordecic mapping $Q: X \rightarrow Y$ such that

$$
\begin{aligned}
& \|f(x)-D(x)\|_{Y} \\
& = \begin{cases}\frac{\delta \epsilon_{\lambda}}{16384^{\beta}-2^{\alpha \lambda}}\|x\|_{X}^{\lambda}, & \lambda \in\left(0, \frac{14 \beta}{\alpha}\right) \\
\frac{2^{\lambda \alpha} \delta \epsilon_{\lambda}}{16384^{\beta}\left(2^{\alpha \lambda}-16384^{\beta}\right)}\|x\|_{X}^{\lambda}, & \lambda \in\left(\frac{14 \beta}{\alpha}, \infty\right)\end{cases}
\end{aligned}
$$

where 


$$
\begin{aligned}
& \epsilon_{\lambda}=\frac{K^{3}}{2^{\beta}}+K^{2} 1716^{\beta}+2 K^{3} 3003^{\beta}+2002^{\beta} K^{4}\left(2^{\alpha \lambda}+1\right)+1001^{\beta} K^{5}\left(3^{\alpha \lambda}+1\right) \\
& +364^{\beta} K^{6}\left(4^{\alpha \lambda}+1\right)+91^{\beta} K^{7}\left(5^{\alpha \lambda}+1\right)+14^{\beta} K^{8}\left(6^{\alpha \lambda}+1\right)+K^{8}\left(7^{\alpha \lambda}+1\right) \\
& +4\left(\frac{14^{\beta} K^{8}}{87178291200}+\frac{91^{\beta} K^{9}}{6227020800}+\frac{364^{\beta} K^{8}}{958003200}+\frac{1001^{\beta} K^{7}}{239500800}+\frac{2002^{\beta} K^{6}}{87091200}\right. \\
& \left.+\frac{3003^{\beta} K^{5}}{43545600}+\frac{1716^{\beta} K^{4}}{29030400}\right)+4\left(2^{\alpha \lambda}\right)\left(\frac{91^{\beta} K^{10}}{87178291200}+\frac{364^{\beta} K^{9}}{6227020800}\right. \\
& \left.+\frac{1001^{\beta} K^{8}}{958003200}+\frac{2002^{\beta} K^{7}}{239500800}+\frac{3003^{\beta} K^{6}}{87091200}+\frac{1716^{\beta} K^{5}}{43545600}+\frac{K^{11}}{58060800}\right) \\
& +4\left(3^{\alpha \lambda}\right)\left(\frac{364^{\beta} K^{10}}{87178291200}+\frac{1001^{\beta} K^{9}}{6227020800}+\frac{2002^{\beta} K^{8}}{958003200}+\frac{3003^{\beta} K^{7}}{239500800}+\frac{1716^{\beta} K^{6}}{87091200}\right) \\
& +4\left(4^{\alpha \lambda}\right)\left(\frac{1001^{\beta} K^{10}}{87178291200}+\frac{2002^{\beta} K^{9}}{6227020800}+\frac{3003^{\beta} K^{8}}{958003200}+\frac{1716^{\beta} K^{7}}{239500800}+\frac{K^{12}}{87091200}\right) \\
& +4\left(5^{\alpha \lambda}\right)\left(\frac{2002^{\beta} K^{10}}{87178291200}+\frac{3003^{\beta} K^{9}}{6227020800}+\frac{1716^{\beta} K^{8}}{958003200}\right) \\
& +4\left(6^{\alpha \lambda}\right)\left(\frac{3003^{\beta} K^{10}}{87178291200}+\frac{1716^{\beta} K^{9}}{6227020800}+\frac{K^{13}}{174182400}\right)+\frac{4\left(7^{\alpha \lambda}\right) 1716^{\beta} K^{10}}{87178291200} \\
& +\frac{4\left(8^{\alpha \lambda}\right) K^{14}}{479001600}+\frac{4\left(10^{\alpha \lambda}\right) K^{15}}{1916006400}+\frac{4\left(12^{\alpha \lambda}\right) K^{16}}{12454041600}+\frac{4\left(14^{\alpha \lambda}\right) K^{17}}{174356582400}
\end{aligned}
$$

The following example shows that the assumption $\lambda \neq \frac{14 \beta}{\alpha}$ cannot be omitted in Corollary 4.2. This example is a modification of well known example of Gajda [6] for the additive functional inequality.

Example 1. Let $\phi: R \rightarrow R$ be defined by

$$
\phi(x)= \begin{cases}x^{14}, & \text { for }|x|<1 \\ 1, & \text { for }|x| \geq 1 .\end{cases}
$$

consider the function $f: R \rightarrow R$ to be defined by

$$
f(x)=\sum_{n=0}^{\infty} 4^{14 n} \phi\left(4^{n} x\right) \quad \forall x \in \mathbb{R} .
$$

Then $f$ satisfies the following functional inequality

$$
|D f(x, y)| \leq \frac{(87178307584) \times(268435456)^{3}}{268435455}\left(|x|^{14}+|y|^{14}\right) \quad \forall x, y \in \mathbb{R} .
$$

Proof. It is easy to see that $f$ is bounded by $\frac{268435456}{268435455}$ on $\mathbb{R}$. If $|x|^{14}+|y|^{14}=0$ or $|x|^{14}+|y|^{14} \geq \frac{1}{268435456}$, then 


$$
\begin{aligned}
|D f(x, y)| & \leq \frac{(87178307584) \times(268435456)}{268435455} \\
& \leq \frac{(87178307584) \times(268435456)^{3}}{268435455}\left(|x|^{14}+|y|^{14}\right)
\end{aligned}
$$

for all $x, y \in \mathbb{R}$. Now, suppose that $0<|x|^{14}+|y|^{14}<\frac{1}{268435456}$. Then there exists a non-negative integer $k$ such that

$$
\frac{1}{(268435456)^{k+2}} \leq|x|^{14}+|y|^{14}<\frac{1}{(268435456)^{k+1}} .
$$

Hence

$$
(268435456)^{K}|x|^{14}<\frac{1}{268435456},(268435456)^{K}|y|^{14}<\frac{1}{268435456}
$$

and

$$
\begin{aligned}
& 4^{n}(x+7 y), 4^{n}(x+6 y), 4^{n}(x+5 y), 4^{n}(x+4 y), 4^{n}(x+3 y), 4^{n}(x+2 y), \\
& 4^{n}(x+y), 4^{n}(x-y), 4^{n}(x-2 y), 4^{n}(x-3 y), 4^{n}(x-4 y), 4^{n}(x-5 y), \\
& 4^{n}(x-6 y), 4^{n}(x-7 y), 4^{n}(x), 4^{n}(y) \in(-1,1) \text { for all } n=0,1, \cdots, k-1 .
\end{aligned}
$$

Hence $D \phi\left(4^{n} x, 4^{n} y\right)=0$ for all $n=0,1, \cdots, k-1$. From the definition of $f$ and the inequality (58), we obtain that

$$
\begin{aligned}
|D f(x, y)| & \leq \sum_{n=k}^{\infty} 4^{-14 n} \cdot(87178307584) \\
& =\frac{(87178307584) \cdot 4^{14(1-k)}}{268435455} \\
& \leq \frac{(87178307584)(268435456)^{3}}{268435455}\left(|x|^{14}+|y|^{14}\right) .
\end{aligned}
$$

Therefore, $f$ satisfies (57) for all $x, y \in \mathbb{R}$. Now, we claim that functional Equation (1) is not stable for $\lambda=14$ in above Corollary (4.2) $(\alpha=\beta=p=1)$.

Suppose on the contrary that there exists a Quattuordecic mapping $Q: \mathbb{R} \rightarrow \mathbb{R}$ and constant $d>0$ such that $|f(x)-Q(x)| \leq d|x|^{14} \forall x \in \mathbb{R}$. Then there exists a constant $c \in \mathbb{R}$ such that $Q(x)=c x^{14}$ for all rational numbers $x$ (see (25)). So we obtain the following inequality

$$
|f(x)| \leq(d+|c|)|x|^{14}, \quad \text { forall } x \in \mathbb{Q} .
$$

Let $m \in \mathbb{N}$ with $m+1>d+|c|$. If $x$ is a rational number in $\left(0,4^{-m}\right)$, then $4^{n} x \in(0,1)$ for all $n=0,1,2, \cdots, m$ and in this case we get

$$
f(x)=\sum_{n=0}^{\infty} \frac{\varphi\left(4^{n} x\right)}{4^{14 n}} \geq \sum_{n=0}^{\infty} \frac{\left(4^{n} x\right)^{14}}{4^{14 n}}=(m+1) x^{14}>(d+|c|)|x|^{14}
$$

which contradicts the inequality (59).

\section{References}

[1] Ulam, S.M. (1960) A Collection of Mathematical Problems. Interscience Publ., New York. 
[2] Hyers, D.H. (1941) On the Stability of the Linear Functional Equation. Proceedings of the National Academy of Sciences of the United States of America, 27, 222-224. https://doi.org/10.1073/pnas.27.4.222

[3] Rassias, T.M. (1978) On the Stability of the Linear Mappings in Banach Spaces. Proceedings of the American Mathematical Society, 72, 297-300. https://doi.org/10.1090/S0002-9939-1978-0507327-1

[4] Aoki, T. (1950) On the Stability of the Linear Transformation in Banach Spaces. Journal of the Mathematical Society of Japan, 2, 64-66. https://doi.org/10.2969/jmsj/00210064

[5] Chang, I.S. and Kim, H.M. (2002) On the Hyers-Ulam Stability of Quadratic Functional Equations. Journal of Inequalities in Pure and Applied Mathematics, 27, Article 33.

[6] Czerwik, S. (2002) Functional Equations and Inequalities in Several Variables. World Scientific, River Edge. https://doi.org/10.1142/4875

[7] Eskandani, G.S., Guvruta, P., Rassias, J.M. and Zarghami, R. (2011) Generalized HyersUlam Stability for a General Mixed Functional Equation in Quasi-Beta Normed Spaces. Mediterranean Journal of Mathematics, 8, 331-348. https://doi.org/10.1007/s00009-010-0082-8

[8] Guvruta, P. (1999) An Answer to a Question of J. M. Rassias Concerning the Sability of Cauchy Functional Equation. In: Advances in Equations and Inequalities, Hardronic Math. Ser., Hadronic Press, Palm Harbor, 67-71.

[9] Guvruta, P. (2001) On a Problem of G. Issac and Th. M. Rassias Concerning the Stability of Mappings. Journal of Mathematical Analysis and Applications, 261, 543-553.

https://doi.org/10.1006/jmaa.2001.7539

[10] Hyers, D.H., Issac, G. and Rassias, T.M. (1998) Stability of Functional Equations in Several Variables. Birkhauser, Basel. https://doi.org/10.1007/978-1-4612-1790-9

[11] Jung, S.M. (2001) Hyers-Ulam-Rassias Stability of Functional Equations in Mathematical Analysis. Hadronic Press, Palm Harbor.

[12] Kannapan, P. (1995) Quadratic Functional Equation and Inner Product Spaces. Results in Mathematics, 27, 368-372. https://doi.org/10.1007/BF03322841

[13] Rassias, J.M. (1984) On Approximately of Approximately Linear Mappings by Linear Mappings. Bulletin des Sciences Mathématiques, 108, 445-446.

[14] Rassias, J.M. and Kim, H.M. (2009) Generalized Hyers-Ulam Stability for General Additive Functional Equation in Quasi Beta Normed Spaces. Journal of Mathematical Analysis and Applications, 356, 302-309. https://doi.org/10.1016/j.jmaa.2009.03.005

[15] Ravi, K., Narasimman, P. and Kishore Kumar, R. (2009) Generalized Hyers-Ulam-Rassias Stability and J. M. Rassias Stability of a Quadratic Functional Equation. International Journal of Mathematical Sciences and Industrial Applications, 3, 79-94.

[16] Ravi, K., Kodandan, R. and Narasimman, P. (2009) Ulam Stability of a Quadratic Functional Equations. International Journal of Pure and Applied Mathematics, 51, 87-101.

[17] Xu, T., Rassias, J.M. and Xu, W.X. (2011) A Fixed Point Approach to the Stability of a General Mixed Type Additive-Cubic Functional Equation in Quasi Fuzzy Normed Spaces. International Journal of Physical Sciences, 6, 313-324.

[18] Xu, T., Rassias, J.M. and Xu, W.X. (2010) A Fixed Point Approach to the Stability of Quintic and Sextic Functional Equations in Quasi- $\beta$ Normed Spaces. Journal of Inequalities and Applications, 2010, Article ID: 423231. https://doi.org/10.1155/2010/423231

[19] Rassias, J.M. and Eslamian, M. (2015) Fixed Point and Stability of Nonic Functional Equation in Quasi-Beta Normed Spaces. Contemporary Analysis and Applied Mathematics, 3, 
293-309. https://doi.org/10.18532/caam.38853

[20] Ravi, K., Rassias, J.M., Pinelas, S. and Sabarinathan, S. (2015) A Fixed Point Approach to the Stability of Decic Functional Equation in Quasi-Beta Normed Spaces. Panamerican Mathematical Journal, 25, 42-52.

\section{Submit or recommend next manuscript to SCIRP and we will provide best service} for you:

Accepting pre-submission inquiries through Email, Facebook, LinkedIn, Twitter, etc. A wide selection of journals (inclusive of 9 subjects, more than 200 journals)

Providing 24-hour high-quality service

User-friendly online submission system

Fair and swift peer-review system

Efficient typesetting and proofreading procedure

Display of the result of downloads and visits, as well as the number of cited articles

Maximum dissemination of your research work

Submit your manuscript at: http://papersubmission.scirp.org/

Or contact apm@scirp.org 